\title{
Effect on Growth, Photosynthesis, and Oxidative Stress of Single Walled Carbon Nanotubes Exposure to Marine Alga Dunaliella tertiolecta
}

\author{
Megha Thakkar, Somenath Mitra, and Liping Wei \\ Department of Chemistry and Environmental Science, New Jersey Institute of Technology, Newark, NJ, USA \\ Correspondence should be addressed to Somenath Mitra; somenath.mitra@njit.edu and Liping Wei; liping.wei@njit.edu
}

Received 28 June 2016; Revised 30 August 2016; Accepted 31 August 2016

Academic Editor: Xingmao Ma

Copyright @ 2016 Megha Thakkar et al. This is an open access article distributed under the Creative Commons Attribution License, which permits unrestricted use, distribution, and reproduction in any medium, provided the original work is properly cited.

Single walled carbon nanotubes were carboxylated by microwave assisted acid oxidation (f-SWCNTs) and examined for their ecotoxicity on marine alga chlorophyte Dunaliella tertiolecta. Toxicity was evaluated based on growth, photosynthetic activities, oxidative stress, and intracellular glutathione in the concentration range of $0.1-20 \mathrm{mg} / \mathrm{L} \mathrm{f}$-SWCNT. Physical interactions between the f-SWCNT and alga were examined using light microscopy and scanning electron microscope. Increasing the nanotube concentration increased the toxic effects where growth inhibition was as high as $30 \%$, photosynthetic yield decreased by as much as $18 \%$, and intracellular glutathione reduction reached $95 \%$. The results from f-SWCNTs were somewhat different when compared to our previous study using the same algae and functionalized multiwalled carbon nanotubes, where exposure led to longer lag phase and higher growth rate inhibition.

\section{Introduction}

Algae present on surface waters are the dominant primary producers that convert inorganic carbon into organic matter in ecosystems [1]. They form a base for the oceanic food web and serve as major components of the global carbon and biogeochemical cycles. Algal populations are affected by anthropogenic pollutants flowing into ecosystems and are important indicators for environment pollution. Nanoparticles with their high surface area and abundant reactive sites can be significant sources of environmental pollution [2]. Algae are an important indicator for environmental pollution monitoring and are widely used as a model organism in ecotoxicity studies of nanomaterials. Algal cell walls act as primary sites for interaction with nanoparticles [3].

Due to the extraordinary physical, chemical, and electronic properties, the commercial production and use of carbon nanotubes (CNTs) have increased rapidly [3, 4]. Worldwide commercial interest in carbon nanotubes is rapidly increasing due to applications in plastics, composites, paints, batteries, touch screens, and drug delivery [5]. CNT release is expected during manufacturing, use, or disposal. Like all other pollutants, CNTs are expected to end up in soil, water, or air [6]. This increases the chance of release into the environment leading to human and ecological risk [7]. CNTs represent a wide range of tubes with different dimensions as well as functionality. Toxicity of multiwalled carbon nanotubes (MWCNTs), single walled carbon nanotubes (SWCNTs), and double walled carbon nanotubes (DWCNTs) has been reported $[8,9]$. Influence of MWCNT on fresh water green algae Chlorella pyrenodisa [3], Chlorella vulgaris [3], and Chlorella sp. $[10,11]$ has been studied. Impact of SWCNT on Raphidocelis subcapitata, Chlorella vulgaris [12], Pseudokirchneriella subcapitata [13], Chromochloris zofingiensis [14], and Scenedesmus obliquus algae [15] has been reported. Toxicity of CNTs to algae has been mainly attributed to agglomeration and physical interaction with cells and attenuation of photosynthesis and CNT induced generation of intracellular reactive oxygen species [3]. However, given the range of CNT based nanoparticles that can make their way to the environment, there is limited knowledge related to the diversity of possible effects on algal functions.

The SWCNTs and MWCNTs have different geometric structures and hence exhibit different cytotoxicity and bioactivity [16]. While there has been several studies with MWCNTs $[3,11,17]$, studies on SWCNTs have been limited. 
Another important consideration is that not many studies have reported the toxicity difference between different types of CNTs using the same marine species and under similar conditions. A recent study with fresh water alga Scenedesmus obliquus showed marked difference in levels of toxicity [15] between single and double walled CNTs, and another study using fresh water algae Pseudokirchneriella subcapitata showed different behavior between metallic and semiconducting SWCNTs [18]. Effects of SWCNT on marine algae, where high salt concentration leads to aggregation, are not well understood, and the effects of SWCNT and MWCNT on the same marine algae and under similar conditions are yet to be studied. Therefore, the differences between MWCNT and SWCNT are not clearly understood. D. tertiolecta is a unicellular, motile, marine green flagellate with size range of 5-10 $\mu \mathrm{m}$.

The objective of this research is to study the effect of oxidized SWCNTs on photosynthesis activity, growth, and oxidative stress using the marine algae $D$. tertiolecta. Since we have published the effect of MWCNT on the same alga [19], another objective of this paper is to elucidate the difference between these two types of CNTs as applied to algal ecotoxicity.

\section{Material and Methods}

2.1. Preparation and Characterization of Carbon Materials. Pristine SWCNTs were obtained from Cheap Tubes Inc. (Cambridgeport, VT, USA). Preweighed amounts of purified SWCNTs were mixed with concentrated 1:1 mixture of $\mathrm{H}_{2} \mathrm{SO}_{4}$ and $\mathrm{HNO}_{3}$ solution in a reactor and treated in a Microwave Accelerated Reaction System (CEM Mars, NC, USA) at $120^{\circ} \mathrm{C}$ for 3 and $10 \mathrm{~min}$, respectively, to produce different oxidation levels; these are designated as f-SWCNT$A$ and $\mathrm{f}-\mathrm{SWCNT}-\mathrm{B}$, respectively. The product was cooled to room temperature, diluted with DI water, and dialyzed until pH is neutral (dialysis bag nominal molecular weight cut-off 12,000-14,000 Daltons). The dialyzed SWCNTs were filtered and dried overnight at $60^{\circ} \mathrm{C}$ under vacuum and were suspended in Milli-Q water at $0.5 \mathrm{mg} / \mathrm{mL}$. The suspension was stable and homogenous. Carbon black was used for comparison and its suspension (Cabot Regal 600 A69, Cabot Corporation, Georgia, United States) was prepared by adding a known amount to Milli-Q at a concentration of $0.5 \mathrm{mg} / \mathrm{mL}$. This suspension was sonicated for 30 minutes but was not stable. Aliquots of the stock suspension were air-dried on silicon wafer and analyzed with a field emission scanning electron microscopy with EDX detector (FESEM-EDX, LEO 1530VP, Oberkochen, Germany).

2.2. Algal Strain and Culturing. Unicellular green algae D. tertiolecta (CCMP 1320) was obtained from Provasoli-Guillard National Center for Marine Algae and Microbiota (NCMA), Bigelow Laboratory for Ocean Science, Maine, USA. D. tertiolecta was maintained in synthetic ocean water (SOW) media Aquil without silicate addition [20]. Polycarbonate bottles (VWR Nalgene, $250 \mathrm{~mL}$, cleaned with detergent and acid) were used for maintenance and culturing. The cultures were incubated in a diurnal growth chamber at $19 \pm 1^{\circ} \mathrm{C}$ with $12 \mathrm{~h}: 12 \mathrm{~h}$ light: dark cycle and $120 \mu \mathrm{mol}$ photons $\mathrm{m}^{-2} \mathrm{~s}^{-1}$ illumination from cool-white fluorescence bulbs.

2.3. Exposure Studies. D. tertiolecta was exposed to different carbon materials-carbon black (CB), f-SWCNT-A, and fSWCNT-B. Test media were prepared by sonicating the $0.5 \mathrm{mg} / \mathrm{mL}$ of stock suspension (f-SWCNT-A, f-SWCNT-B, and $\mathrm{CB}$ ) prior to addition to the culture media Aquil to get nominal concentrations of $0,0.1,1,2.5,5,10$, and $20 \mathrm{mg} / \mathrm{L}$. These media were allowed to preequilibrate for 24 -hr on a horizontal shaker (120 rpm). D. tertiolecta of late exponential phase was added at $1: 100 \mathrm{v}: \mathrm{v}$ ratio of inoculums to media. Polycarbonate bottles (125 mL, VWR Nalgene) and borosilicate culture tubes ( $5 \mathrm{~mL}$, VWR 47729-570) used in this study were precleaned with detergent and acid. The cultures were put on a horizontal shaker $(120 \mathrm{rpm})$ and incubated in the growth chamber. Cultures were set up with 3-4 replicates. Additional tests were done by filtering f-SWCNT-A using $0.2 \mu \mathrm{m}$ PTFE filters to remove the aggregates. The filtrate was then tested for toxicity. Throughout the exposure study, control cultures without the carbon material and blanks (with carbon materials but without algae inoculation) were used to test for interference and agglomeration.

2.4. Culture Analysis. Since the exposure to the SWCNTs might induce oxidative stress, cause growth inhibition, and alter photosynthetic functions, the culture analyses focused on observable endpoints for growth, photosynthesis function, and oxidative stress.

Algal growth was evaluated with in vivo fluorescence, exponential growth rate, and total chlorophyll a. Specifically in vivo fluorescence (IVF) was measured on daily basis using Laboratory Fluorometer (Trilogy, Turner Design, CA, USA) equipped with an optical block of $485 \mathrm{~nm}$ excitation and $685 \mathrm{~nm}$ emission with a $50 \mathrm{~nm}$ bandwidth. The exponential growth rate was obtained from linear regression of logarithmic transformation of in vivo fluorescence (LN (IVF)) over time. In addition, total chlorophyll a ( $\mathrm{Chl}$ a) and cell density were quantified. Total chlorophyll a concentrations were estimated by acetone ( $90 \%$ acetone and $10 \%$ water mixture) extraction of pigments collected from $50 \mathrm{~mL}$ of the culture on a $25 \mathrm{~mm}$ GF/F filter. This was followed by Jeffery and Humphrey's trichromatic quantification using Agilent 8453 spectrophotometer following EPA Method 446.0 [21]. Cell density of $D$. tertiolecta was determined using coulter counter equipped with a $70 \mu \mathrm{m}$ aperture tube (Beckman Coulter, Multisizer 3). The particles in the size window 5.2-9.6 $\mu \mathrm{m}$ were predominately $D$. tertiolecta cells, while those of size 1.7$5.2 \mu \mathrm{m}$ were from agglomerated CNTs. Measurements were made at a concentration of $20 \mathrm{mg} / \mathrm{L}$.

Scanning electron microscopy (SEM) and light microscope (with immersion oil) were used to view the surface interaction between cells and the f-SWCNTs. For SEM, the cells exposed to f-SWCNTs were filtered onto $0.2 \mu \mathrm{m}$ polycarbonate filter, rinsed with MQ water, transferred onto silicon wafer, and air-dried. The samples were carbon-coated (BalTEC 020 HR Sputtering coater) and viewed under scanning electron microscope. 


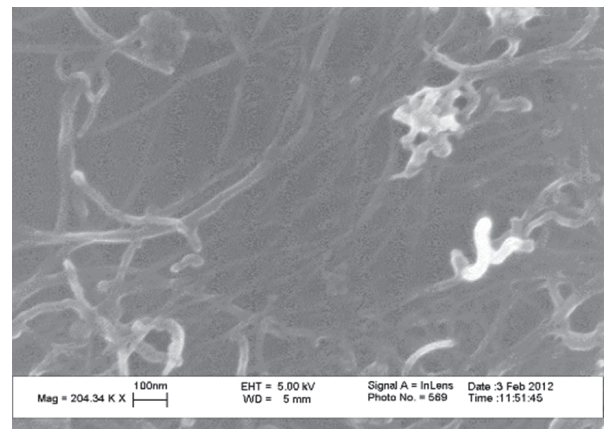

(a)

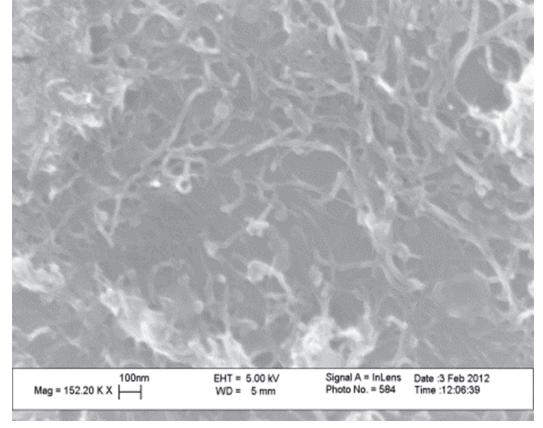

(b)

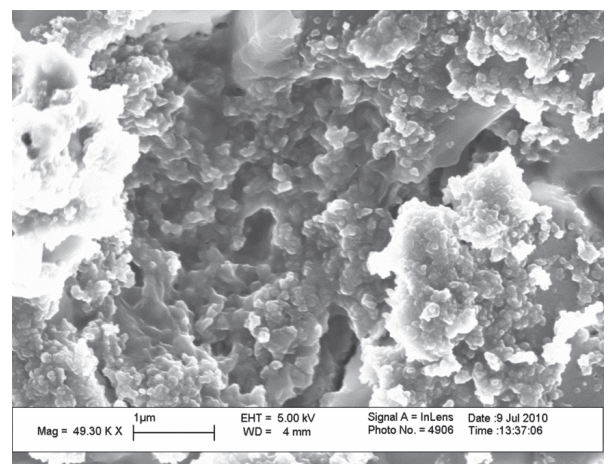

(c)

FIGURE 1: Scanning electron microscopy images of (a) f-SWCNT-A; (b) f-SWCNT-B; and (c) carbon black.

2.5. Photosynthesis. Algal photochemical function was evaluated using photosystem II (PSII). The photochemical parameters were recorded on a daily basis during mid-exponential growth phase to late exponential growth phase by subjecting aliquots of the cultures to dark-adaption (15-min) followed by fluorescence induction and relaxation characterization using Satlantic FIRe System (Satlantic, Inc.) under single turnover flash protocol [22]. The FIRe measurement consisted of induction phase and relaxation phase. In the induction phase a short pulse of 100 seconds (called single turnover flash) was applied to cumulatively saturate photosystem II (PSII) and the fluorescence induction kinetics from F0 (minimum fluorescence) to Fm (maximum fluorescence) were measured. In the relaxation phase a weak modulated light was applied to record the relaxation kinetics of fluorescence yields on the timescale of $500 \mathrm{~ms}$. The FIRe data were processed with the instrument's software FIRe Pro (version 1.3.1) to obtain various parameters describing PSII photochemical processes.

2.6. Glutathione. Total glutathione was quantified for algal cells to evaluate the oxidative stress that might be induced by the exposure to f-SWCNTs. Total glutathione was determined following procedure reported before [19]. Known aliquots of the algal cultures were filtered on GF/F filters. The collected algal cells were heated in $10 \mathrm{mM}$ methanesulfonic acid at $70^{\circ} \mathrm{C}$ for $2 \mathrm{~min}$ and then sonicated (Fisher Scientific FS-28) in ice-cold water for $30 \mathrm{~min}$. This led to the cell lysis and the extraction of cellular thiols. The thiols were reduced and tagged with bimane by reacting with excessive amount of monobromobimane at $\mathrm{pH}$ 9. The reaction was terminated upon acidification with methanesulfonic acid solution. The thiol-bimane adduct was analyzed by HPLC with fluorescence detector following a previously published method [19]. The quantified glutathione was further normalized to chlorophyll a.

The experimental data were analyzed by analysis of variance (ANOVA) using MINITAB 16 statistical analysis. Probability $p<0.05$ was accepted as statistically significant. When needed, Tukey test with family error rate of $5 \%$ was also used.

\section{Results and Discussion}

The pristine single walled carbon nanotubes had a length of $5-30 \mu \mathrm{m}$ and outer diameter of $1.1 \mathrm{~nm}$. Figure 1 shows the scanning electron microscopy (SEM) images of the SWCNTs used in this study; f-SWCNT-A (Figure 1(a)) and f-SWCNT-B (Figure 1(b)) showed similar tubular structures. As expected, carbon black sample (Figure 1(c)) did not show the presence of any tubes. The ratio of $\mathrm{C}: \mathrm{O}:$ Fe weight percent measured by SEM-EDX for f- SWCNT-A was $82: 17: 1$ and for $\mathrm{f}$ SWCNT-B was $75: 24: 1$.

3.1. Effect of SWCNTs on Algal Growth. Exponentially growing $D$. tertiolecta cells were inoculated into the preequilibrated media containing 0.1 to $20 \mathrm{mg} / \mathrm{L}$ of the sample to be studied. The resulting growth curves are shown in Figure 2. It is seen that increasing $\mathrm{f}-\mathrm{SWCNT}$ concentration resulted in 


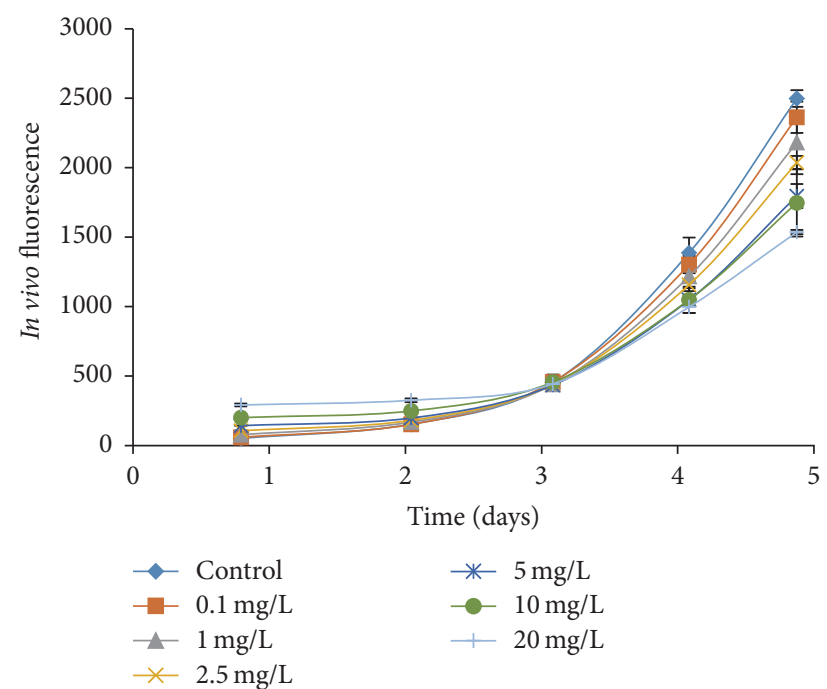

(a)

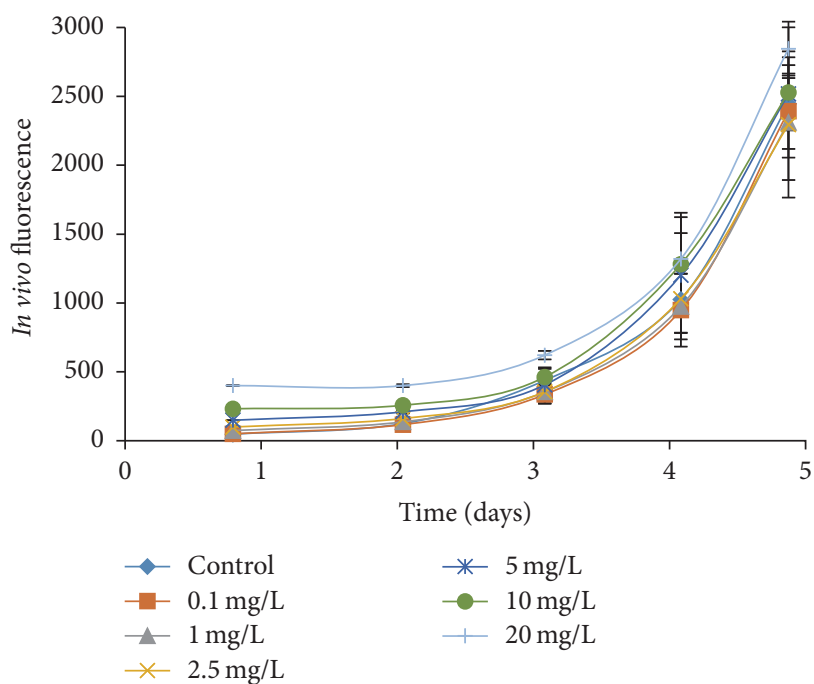

(c)

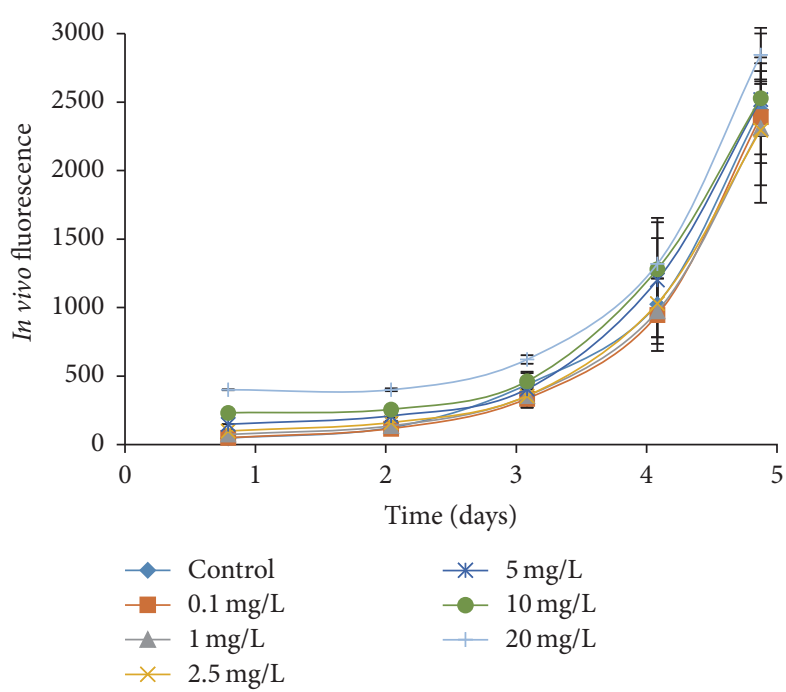

(b)

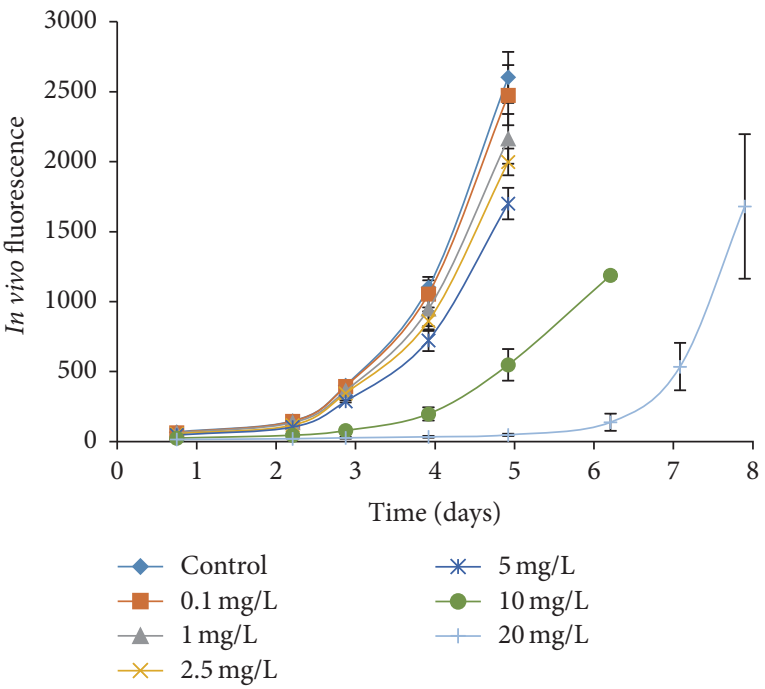

(d)

Figure 2: In vivo chlorophyll a fluorescence based growth curves of D. tertiolecta exposed to 0, 0.1, 1, 2.5, 5, 10, and $20 \mathrm{mg} / \mathrm{L}$ of samples: (a) f-SWCNT-A; (b) filtrate; (c) f- SWCNT-B; and (d) carbon black.

increased growth inhibition. When alga was exposed to 10 and $20 \mathrm{mg} / \mathrm{L}$ of carbon black it showed a lag phase of 3 days and 4 days, respectively. Cells exposed to the two SWCNTs did not show any lag phase. The SWCNT was quite different from what was observed with MWCNT with the same alga [19], which showed a 23-day lag phase at CNT concentration of $10 \mathrm{mg} / \mathrm{L}$.

The one-way ANOVA with Tukey test were performed on the growth rate. Concentrations that showed significant effect compared with control are presented in Table 1. Exposure to carbon black did not show any significant growth rate inhibition. On the other hand, both the f-SWCNTs showed significant growth inhibition. Based on 2-sample $t$-test on exponential growth rate, there was no difference between f-SWCNT-A and f-SWCNT-B. However, there was a significant difference between f-SWCNT-A and the filtrate where the nanotube aggregates had been removed. The filtrate containing only the highly dispersed nanotubes showed growth inhibition only at high concentration $(20 \mathrm{mg} / \mathrm{L})$. Thus removal of aggregates by filtration decreased the growth rate inhibition and reduced toxicity. In the present study, exposure to $10 \mathrm{mg} / \mathrm{L}$ of carbon black showed no effect on exponential growth rate and exposure to $10 \mathrm{mg} / \mathrm{L}$ of f-SWCNT-A and f-SWCNT-B showed exponential growth rate inhibition of $22 \pm 3.9 \%$ and $29 \pm 5 \%$, respectively. On a comparative basis, our previous study [19] with f-MWCNTs had shown a growth rate inhibition of $36 \%$ at a concentration of $10 \mathrm{mg} / \mathrm{L}$. Other studies using SWCNT and DWCNT using fresh water algae have shown inhibition as high as 40 to $52 \%[12,15]$.

3.2. Aggregation in Presence of SWCNT. Dynamic light scattering (DLS) was used to measure the particle size of the pure nanocarbons in deionized as well as sea water. Concentrations of $20 \mathrm{mg} / \mathrm{L}$ of carbon black, f-SWCNT-A, and 


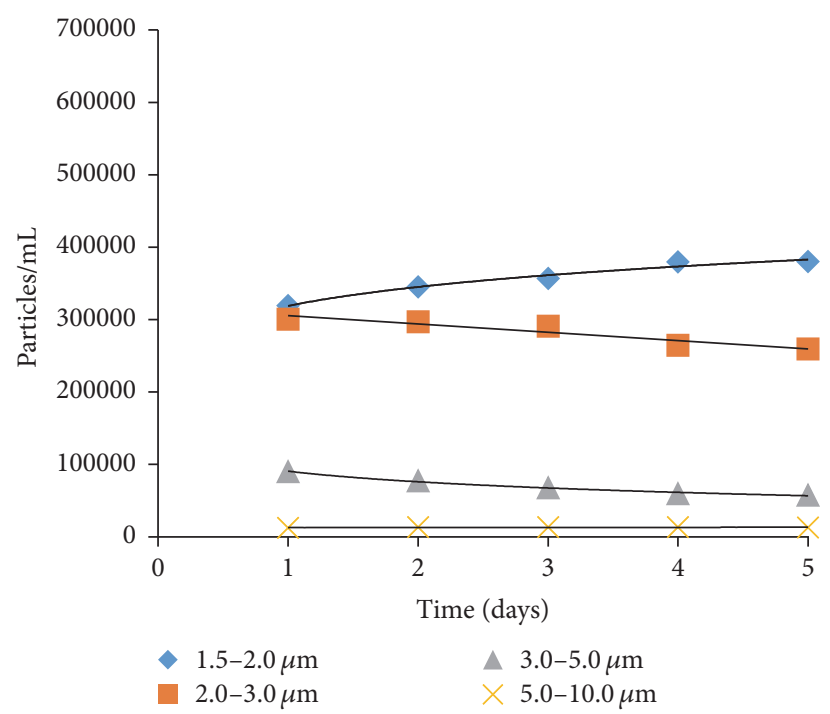

(a)

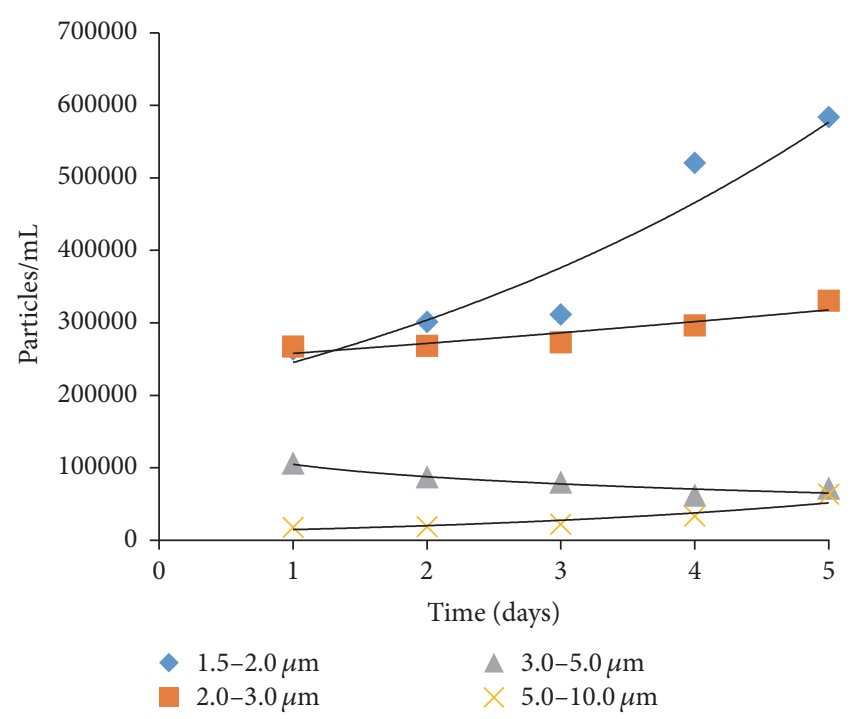

(b)

Figure 3: Particle size measurements using Multisizer coulter counter. (a) f-SWCNT-B without alga. (b) f-SWCNT-B and alga.

TABLE 1: Significant effect on growth rate by one-way ANOVA with Tukey's test.

\begin{tabular}{lccc}
\hline Material & $\begin{array}{c}\text { Concentration } \\
(\mathrm{mg} / \mathrm{L})\end{array}$ & \% of inhibition & $\begin{array}{c}\text { ANOVA } \\
\text { data }^{*}\end{array}$ \\
\hline \multirow{3}{*}{ f-SWCNT-A } & 5 & $17 \pm 1.5$ & $\mathrm{~B}$ \\
& 10 & $22 \pm 3.9$ & $\mathrm{C}$ \\
\hline f-SWCNT-filtrate & 20 & $27 \pm 2.4$ & $\mathrm{C}$ \\
\hline \multirow{2}{*}{ f-SWCNT-B } & 5 & $12 \pm 2.4$ & $\mathrm{~B}$ \\
& 10 & $18 \pm 2.8$ & $\mathrm{~B}$ \\
& 20 & $29 \pm 5.0$ & $\mathrm{C}$ \\
\hline
\end{tabular}

*ANOVA analysis of control and unaffected were indicated with alphabet A while B, C, and D represent significant effect.

f-SWCNT-B were prepared in Milli-Q and synthetic ocean water (SOW) to measure particle size and zeta potential. Samples prepared in Milli-Q had a polydispersity of $<0.5$, average size of carbon black was $91 \pm 1 \mathrm{~nm}$, f-SWCNT-A was $114.5 \pm 9 \mathrm{~nm}$, and $\mathrm{f}-$ SWCNT-B was $165.5 \pm 4 \mathrm{~nm}$. Zeta potential of SWCNT was measured by diluting the stock solution to $5 \mathrm{mg} / \mathrm{L}$ with Milli-Q and SOW. Both f-SWCNT$A$ and f-SWCNT-B were stable in Milli-Q with zeta potential of $-39.4 \mathrm{mV}$ and $-33.4 \mathrm{mV}$, respectively.

When the suspension was prepared in SOW, the nanotubes were unstable due to aggregation and had a zeta potential of $-8.4 \mathrm{mV}$ and $-7.4 \mathrm{mV}$ for f-SWCNT-A and $\mathrm{f}-$ SWCNT-B, respectively. High concentrations of cationic ions $\mathrm{Ca}^{2+}, \mathrm{Mg}^{2+}$, and $\mathrm{Na}^{2+}$ in seawater are known to destabilize carboxylated CNT suspension by compressing the electrical double layer [23]. Carbon black had an average size of $473 \pm 67 \mathrm{~nm}$; f-SWCNT-A and f-SWCNT-B showed similar trimodal size distribution with peaks around 100, 450, and $750 \mathrm{~nm}$.
Particle in presence of nanocarbons and algae was monitored using the coulter counter. The f-SWCNT-B exposed cells were monitored daily using $70 \mu \mathrm{m}$ aperture tube and particles in the range of 1.7 to $10 \mu \mathrm{m}$ were quantified. Numbers of particles at different size ranges, 1.7-2, 2-3, 3-5, and 5$10 \mu \mathrm{m}$, were monitored, and these are shown in Figures 3(a) and 3(b). D. tertiolecta represented size range of 5.2-9.6 $\mu \mathrm{m}$, and the nanocarbons represented the smaller diameters. It was seen that the smaller particles were more abundant. Figure 3(a) represents the nanocarbon blank without the alga. It is seen that the particles in the different size ranges did not change dramatically with time; the particles between $1.7-2,2-3,3-5$, and $5-10 \mu \mathrm{m}$ increased by $19,14,36$, and $3 \%$, respectively. On the other hand, in presence of algae (Figure 3(b)) the corresponding changes for the same size ranges were $121,24,31$, and $259 \%$, respectively. The increase in concentration of the small particle in presence of the alga is attributed to organic matter secreted from algal cells which led to further aggregation. Similar results were observed in our previous study with exposure to f- MWCNT [19] and by Schwab et al. 2011 [24].

Physical interactions of f-SWCNT-B with alga were also directly observed using optical microscopy and scanning electron microscopy (Figures 4 and 5). Light microscopy showed presence of active cells with SWCNT aggregates on the surface and nonmotile cell entrapped in layers of agglomerated SWCNT. Some cells had also lost cellular integrity and exhibited cytoplasm leakage. Study of single walled carbon nanotubes on $E$. coli has shown damage of cell membrane and leakage of intracellular matter [25]. Scanning electron microscopy shown in Figure 5 also showed the presence of SWCNT aggregates on cell surface.

3.3. Photosynthesis. Photosynthesis was monitored by fluorescence induction and relaxation (FIRe) technique, which is well established approach to study photosynthesis functions 


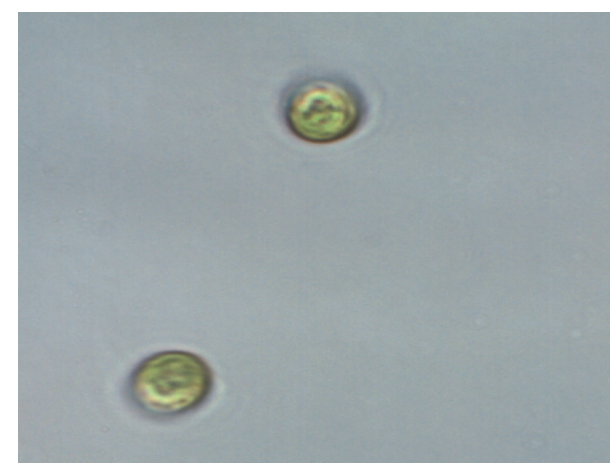

(a)

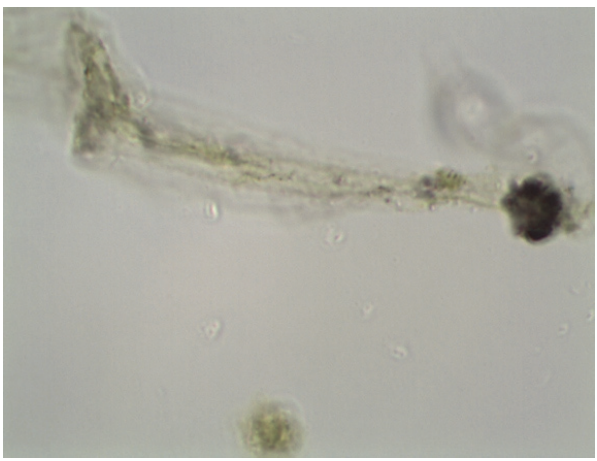

(c)

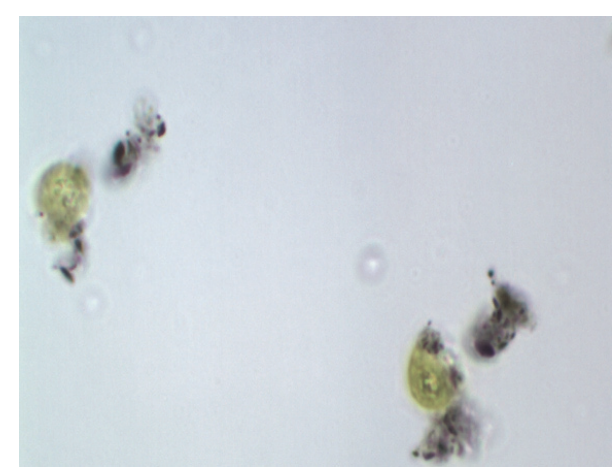

(b)

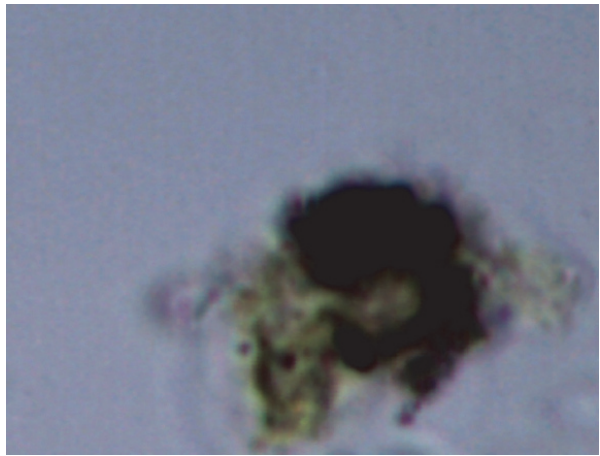

(d)

FIGURE 4: Light microscopy of (a) D. tertiolecta. (b-d) Different cell aggregated with f-SWCNT-B.

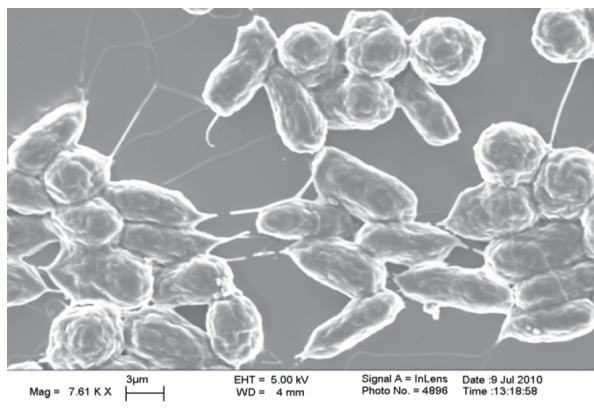

(a)

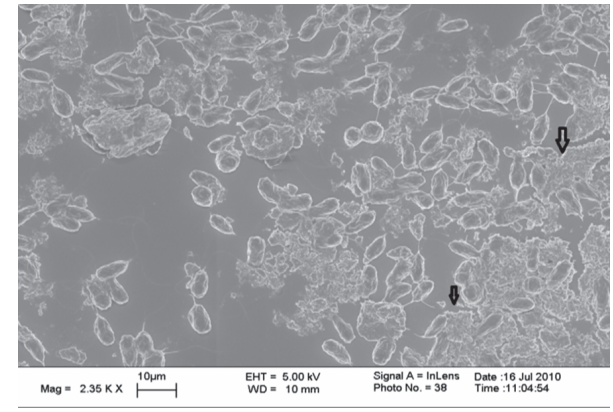

(b)

Figure 5: Scanning electron microscopic images of (a) D. tertiolecta and (b) cells exposed to f-SWCNT-B showing nanotube aggregates on cell surface.

[19]. Induction phase parameters $F_{v} / F_{m}$ were a measure of quantum yield of PSII, $p$ represented the energy transfer between the individual PSII units involved in photosynthesis, and Sigma represents the functional optical cross section of the PSII. The relaxation parameters measure the capability of the reaction center in the photosynthetic units.

The data was analyzed by one-way ANOVA with Tukey test. Concentrations that showed significant effect are presented in Table 2. Cells exposed to f-SWCNT-B showed significant effect on induction parameters such as photosynthetic quantum yield $\left(F_{v} / F_{m}\right)$, Sigma, $p$ value, and Tauav2 (Table 2 ). The quantum yield decreased by $18 \%$ at concentration $20 \mathrm{mg} / \mathrm{L}$. The PSII cross section and connectivity factor p were reduced by 12 and $21 \%$ at 10 and $20 \mathrm{mg} / \mathrm{L}$, respectively. The latter defines the energy transfer between individual PSII units. Increase of Tauav2 by 114 and $116 \%$, respectively, at 10 and $20 \mathrm{mg} / \mathrm{L}$ indicated the presence of small fraction of inactive PSII reaction centers that were incapable of fast electron capture. For cells exposed to filtrate without SWCNT aggregates showed reduction in quantum yield by $22 \%$ at $20 \mathrm{mg} / \mathrm{L}$. The photosynthetic effects for f-SWCNT-B were more or less similar but there were some differences. These are shown in Table 2. The quantum yield decreased by as much as $11 \%$. The results indicate that the presence of f-SWCNTA affected mainly induction parameters while f-SWCNT-B affected the relaxation parameters as well. Our previous study 


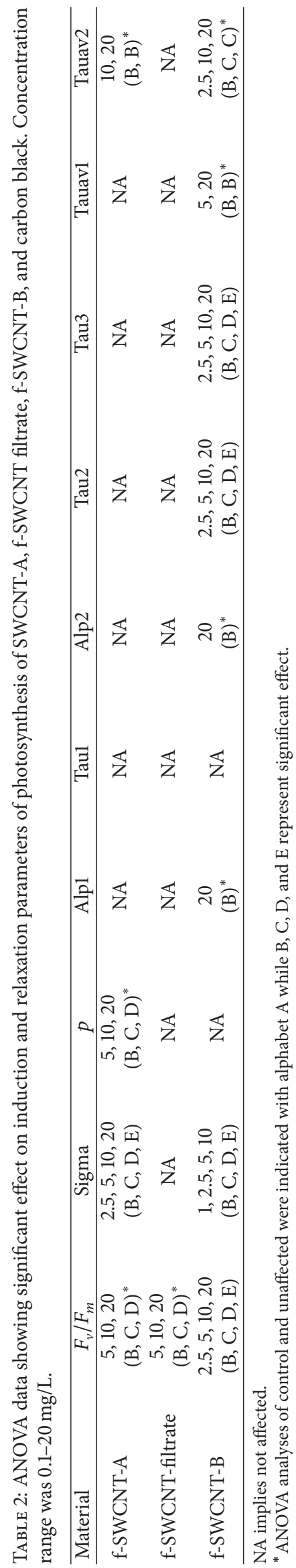




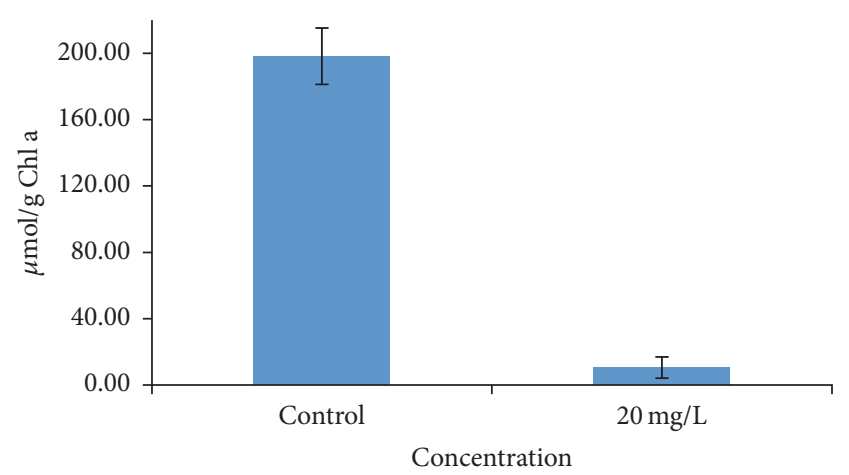

FIGURE 6: Glutathione quantification of D. tertiolecta and cells exposed to $20 \mathrm{mg} / \mathrm{L}$ of $\mathrm{f}-\mathrm{SWCNT}-\mathrm{B}$.

related to the exposure of MWCNT to the same algae showed similar effect on different photosynthetic parameters [19] and this is in line with other studies using fresh water algae [2].

3.4. Oxidative Stress. The source of oxidative stress has been debated. Reactive oxygen species (ROS) production and subsequent damage of cellular components have often been considered as the cause of oxidative stress [26] while others have put forward non-ROS related mechanisms [25, 27]. Many studies have reported oxidative stress as one of the mechanisms for toxicity in different types of algae $[19,24$, 25 , 27]. After $120 \mathrm{hrs}$ exposure with $20 \mathrm{mg} / \mathrm{L}$ f-SWCNT-B, the cells were harvested for glutathione quantification (Figure 6). Compared to the control, exposed cells showed $95 \pm 3 \%$ decrease in total glutathione level when normalized to $\mu$ moles of Chl a (Figure 6). $t$-test indicated $20 \mathrm{mg} / \mathrm{L}$ was significantly different than the control. This was a clear indication of oxidative stress and the absolute value is similar to what was reported before for MWCNTs using the same alga [19].

\section{Conclusion}

It is concluded that f-SWCNTs can induce growth inhibition, cause harm to photosynthetic system, and adversely affect cellular glutathione levels in D. tertiolecta. Majority of the toxicity was attributed to the aggregates of f-SWCNT. Removal of aggregates by filtration decreased the toxicity effects. Exposure to $10 \mathrm{mg} / \mathrm{L}$ of $\mathrm{f}-\mathrm{SWCNT}$ did not show lag phase, whereas carbon black showed a lag phase of 3 days. On comparative basis our previous studies with f-MWCNT had shown a lag phase of 23 days implying that the SWCNTs behave differently from MWCNTs. The photosynthetic activity was comparable to what we had observed with MWCNTs. The presence of SWCNTs also showed a 95\% inhibition in intracellular glutathione concentrations.

\section{Disclosure}

Any opinions, findings, and conclusions or recommendations expressed in this material are those of the author(s) and do not necessarily reflect the views of the NIEHS.

\section{Competing Interests}

The authors declare that there is no conflict of interests regarding the publication of this paper.

\section{Acknowledgments}

This work was funded by the National Institute of Environmental Health Sciences (NIEHS) under Grant no. R01ES023209.

\section{References}

[1] J. Du, S. Wang, H. You, and X. Zhao, "Understanding the toxicity of carbon nanotubes in the environment is crucial to the control of nanomaterials in producing and processing and the assessment of health risk for human: a review," Environmental Toxicology and Pharmacology, vol. 36, no. 2, pp. 451-462, 2013.

[2] D. N. Matorin, A. V. Karateyeva, V. A. Osipov, E. P. Lukashev, N. K. Seifullina, and A. B. Rubin, "Influence of carbon nanotubes on chlorophyll fluorescence parameters of green algae Chlamydomonas reinhardtii," Nanotechnologies in Russia, vol. 5, no. 5-6, pp. 320-327, 2010.

[3] L. Zhang, C. Lei, J. Chen, K. Yang, L. Zhu, and D. Lin, "Effect of natural and synthetic surface coatings on the toxicity of multiwalled carbon nanotubes toward green algae," Carbon, vol. 83, pp. 198-207, 2015.

[4] I. Schwyzer, R. Kaegi, L. Sigg, R. Smajda, A. Magrez, and B. Nowack, "Long-term colloidal stability of 10 carbon nanotube types in the absence/presence of humic acid and calcium," Environmental Pollution, vol. 169, pp. 64-73, 2012.

[5] M. F. L. De Volder, S. H. Tawfick, R. H. Baughman, and A. J. Hart, "Carbon nanotubes: present and future commercial applications," Science, vol. 339, no. 6119, pp. 535-539, 2013.

[6] B. Nowack, R. M. David, H. Fissan et al., "Potential release scenarios for carbon nanotubes used in composites," Environment International, vol. 59, pp. 1-11, 2013.

[7] E. J. Petersen and T. B. Henry, "Methodological considerations for testing the ecotoxicity of carbon nanotubes and fullerenes: review," Environmental Toxicology and Chemistry, vol. 31, no. 1, pp. 60-72, 2012.

[8] P. Jackson, N. R. Jacobsen, A. Baun et al., "Bioaccumulation and ecotoxicity of carbon nanotubes," Chemistry Central Journal, vol. 7, no. 1, article 154, 2013.

[9] W.-C. Hou, P. Westerhoff, and J. D. Posner, "Biological accumulation of engineered nanomaterials: a review of current knowledge," Environmental Sciences: Processes \& Impacts, vol. 15, no. 1, pp. 103-122, 2013.

[10] M. M. Pereira, L. Mouton, C. Yéprémian et al., "Ecotoxicological effects of carbon nanotubes and cellulose nanofibers in Chlorella vulgaris," Journal of Nanobiotechnology, vol. 12, no. 1, article 15, 2014.

[11] Z. Long, J. Ji, K. Yang, D. Lin, and F. Wu, "Systematic and quantitative investigation of the mechanism of carbon nanotubes' toxicity toward Algae," Environmental Science and Technology, vol. 46, no. 15, pp. 8458-8466, 2012.

[12] E. K. Sohn, Y. S. Chung, S. A. Johari et al., "Acute toxicity comparison of single-walled carbon nanotubes in various freshwater organisms," BioMed Research International, vol. 2015, Article ID 323090, 7 pages, 2015. 
[13] S. Youn, R. Wang, J. Gao et al., "Mitigation of the impact of single-walled carbon nanotubes on a freshwater green algae: Pseudokirchneriella subcapitata," Nanotoxicology, vol. 6, no. 2, pp. 161-172, 2012.

[14] Y. Wang and K. Yang, "Toxicity of single-walled carbon nanotubes on green microalga Chromochloris zofingiensis," Chinese Journal of Oceanology and Limnology, vol. 31, no. 2, pp. 306-311, 2013.

[15] F. Mou, P. Wang, H. Li, and Z. Zhou, "Growth inhibitions of four types of CNTs on Scenedesmus obliquus," Journal of Convergence Information Technology, vol. 8, no. 10, pp. 176-182, 2013.

[16] G. Jia, H. Wang, L. Yan et al., "Cytotoxicity of carbon nanomaterials: single-wall nanotube, multi-wall nanotube, and fullerene," Environmental Science \& Technology, vol. 39, no. 5, pp. 13781383, 2005.

[17] R. K. Basniwal, R. P. S. Chauhan, V. Bhatia, and V. K. Jain, "Toxicity study of multiwalled carbon nanotubes on freshwater aquatic algae," Journal of Bionanoscience, vol. 7, no. 5, pp. 597600, 2013.

[18] J. G. Clar, S. A. Gustitus, S. Youn, C. A. Silvera Batista, K. J. Ziegler, and J. C. J. Bonzongo, "Unique toxicological behavior from single-wall carbon nanotubes separated via selective adsorption on hydrogels," Environmental Science \& Technology, vol. 49, no. 6, pp. 3913-3921, 2015.

[19] L. Wei, M. Thakkar, Y. Chen, S. A. Ntim, S. Mitra, and X. Zhang, "Cytotoxicity effects of water dispersible oxidized multiwalled carbon nanotubes on marine alga, Dunaliella tertiolecta," Aquatic Toxicology, vol. 100, no. 2, pp. 194-201, 2010.

[20] N. M. Price, G. I. Harrison, J. G. Hering et al., "Preparation and chemistry of the artificial algal culture medium Aquil," Biological Oceanography, vol. 6, no. 5-6, pp. 443-461, 1989.

[21] E. J. Arar, Method 446.0 in Vitro Determination of Chlorophyll $a, b, c 1+c 2$ and Pheopigments in Marine and Freshwater Algae by Visible Spectrophotometry, National Exposure Research Laboratory Office of Research and Development U.S. Environmental Protection Agency, Cincinnati, Ohio, USA, 1997.

[22] M. Y. Gorbunov and P. G. Falkowski, "Fluorescence induction and relaxation (FIRe) technique and instrumentation for monitoring photosynthetic processes and primary production in aquatic ecosystems," in Photosynthesis: Fundamental Aspects to Global Perspectives. Proc. 13th Int. Congress of Photosynthesis, 29 August-3 September 2004, A. van der Est and D. Bruce, Eds., pp. 1029-1031, Allen Press, Oxford, UK, 2004.

[23] R. D. Handy, R. Owen, and E. Valsami-Jones, "The ecotoxicology of nanoparticles and nanomaterials: current status, knowledge gaps, challenges, and future needs," Ecotoxicology, vol. 17, no. 5, pp. 315-325, 2008.

[24] F. Schwab, T. D. Bucheli, L. P. Lukhele et al., "Are carbon nanotube effects on green algae caused by shading and agglomeration?" Environmental Science and Technology, vol. 45, no. 14, pp. 6136-6144, 2011.

[25] S. Kang, M. Pinault, L. D. Pfefferle, and M. Elimelech, "Singlewalled carbon nanotubes exhibit strong antimicrobial activity," Langmuir, vol. 23, no. 17, pp. 8670-8673, 2007.

[26] Q. Li, S. Mahendra, D. Y. Lyon et al., "Antimicrobial nanomaterials for water disinfection and microbial control: potential applications and implications," Water Research, vol. 42, no. 18, pp. 4591-4602, 2008.

[27] D. Y. Lyon and P. J. J. Alvarez, "Fullerene water suspension $\left(\mathrm{nC}_{60}\right)$ exerts antibacterial effects via ROS-independent protein oxidation," Environmental Science \& Technology, vol. 42, no. 21, pp. 8127-8132, 2008. 

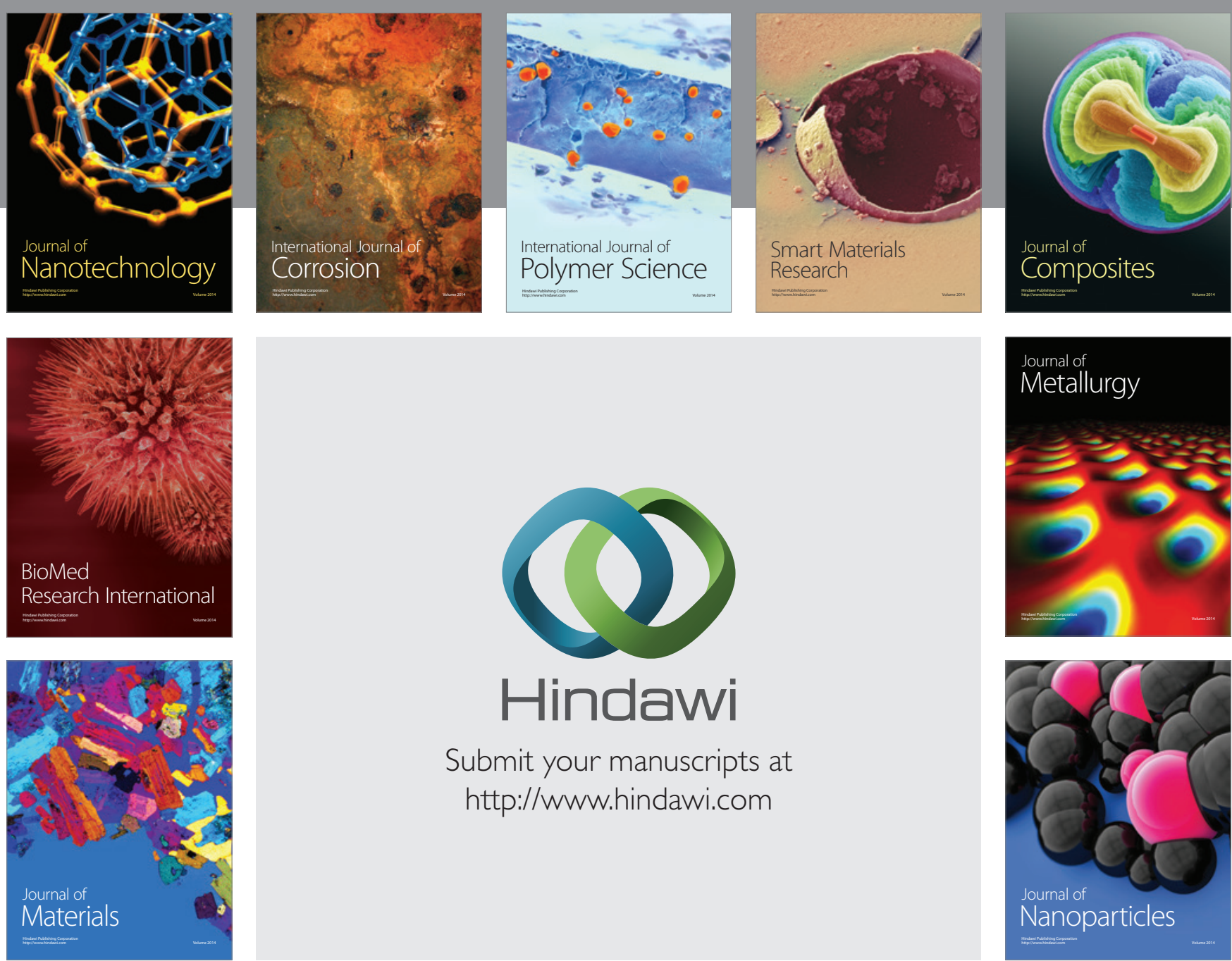

\section{Hindawi}

Submit your manuscripts at

http://www.hindawi.com

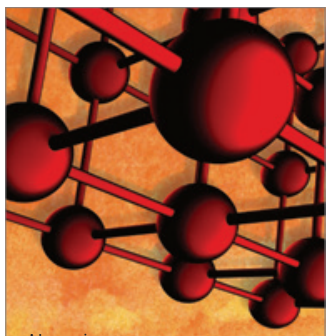

Materials Science and Engineering
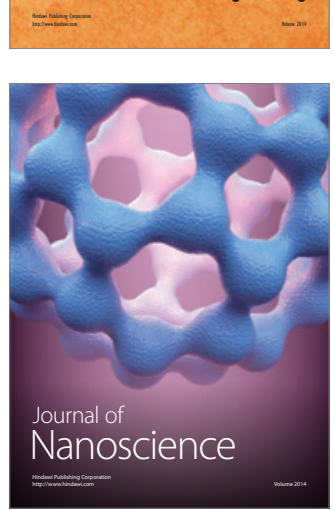
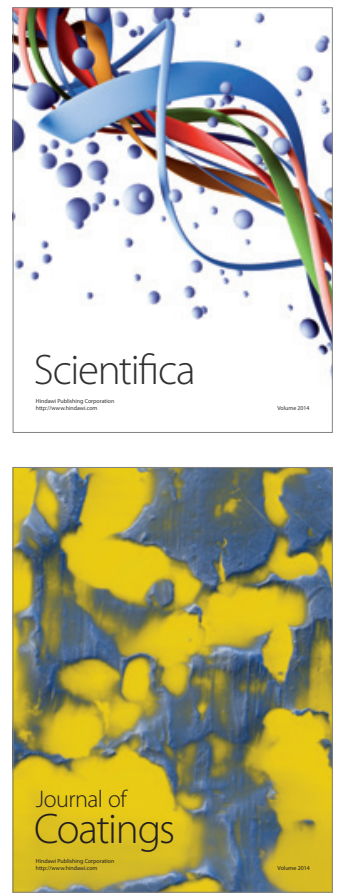
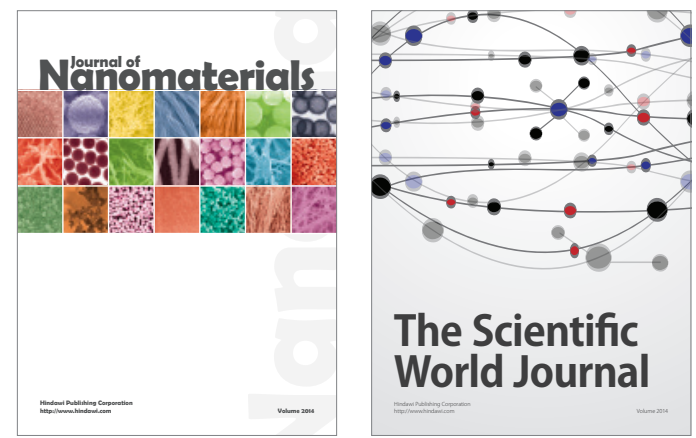

The Scientific World Journal
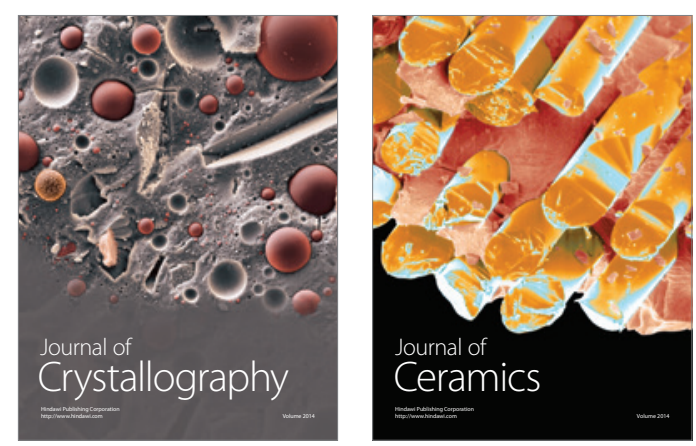
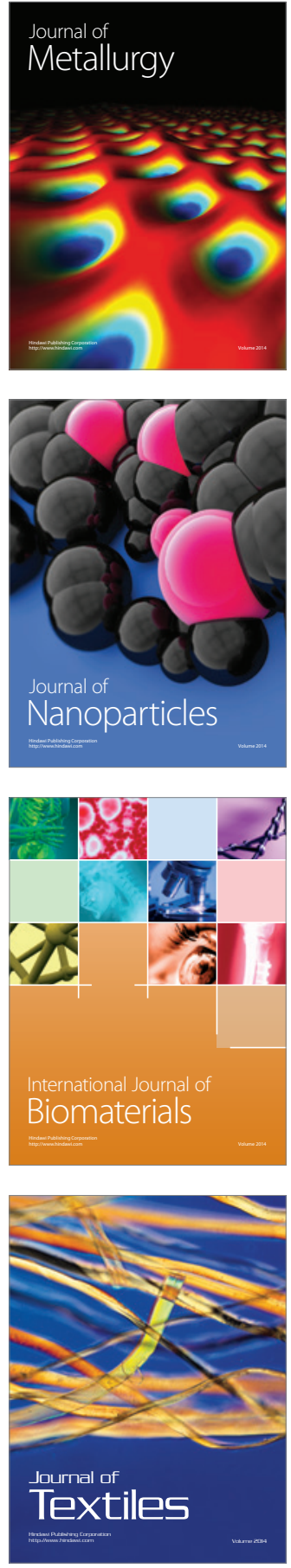